ARTICLE

Received 29 Jan 2015 | Accepted 9 Sep 2015 | Published 20 Oct 2015

DOI: $10.1038 /$ ncomms9586

OPEN

\title{
High-temperature superconductivity in space-charge regions of lanthanum cuprate induced by two-dimensional doping
}

\author{
F. Baiutti ${ }^{1}$, G. Logvenov ${ }^{1}$, G. Gregori ${ }^{1}$, G. Cristiani ${ }^{1}$, Y. Wang ${ }^{2}$, W. Sigle ${ }^{2}$, P.A. van Aken ${ }^{2}$ \& J. Maier ${ }^{1}$
}

The exploitation of interface effects turned out to be a powerful tool for generating exciting material properties. Such properties include magnetism, electronic and ionic transport and even superconductivity. Here, instead of using conventional homogeneous doping to enhance the hole concentration in lanthanum cuprate and achieve superconductivity, we replace single $\mathrm{LaO}$ planes with $\mathrm{SrO}$ dopant planes using atomic-layer-by-layer molecular beam epitaxy (two-dimensional doping). Electron spectroscopy and microscopy, conductivity measurements and zinc tomography reveal such negatively charged interfaces to induce layer-dependent superconductivity ( $T_{c}$ up to $35 \mathrm{~K}$ ) in the space-charge zone at the side of the planes facing the substrate, where the strontium ( $\mathrm{Sr}$ ) profile is abrupt. Owing to the growth conditions, the other side exhibits instead a Sr redistribution resulting in superconductivity due to conventional doping. The present study represents a successful example of two-dimensional doping of superconducting oxide systems and demonstrates its power in this field.

\footnotetext{
${ }^{1}$ Max Planck Institute for Solid State Research, Heisenbergstrasse 1, Stuttgart 70569, Germany. ${ }^{2}$ Max Planck Institute for Intelligent Systems, Heisenbergstrasse 3, Stuttgart 70569, Germany. Correspondence and requests for materials should be addressed to G.L. (email: g.logvenov@fkf.mpg.de) or to G.G. (email: g.gregori@fkf.mpg.de) or to J.M. (email: s.weiglein@fkf.mpg.de).
} 
nterface effects in complex oxide heterostructures have received great attention because of the emergence of functional properties such as magnetism ${ }^{1-4}$, metallicity ${ }^{5-7}$, superconductivity ${ }^{8,9}$ and high-temperature superconductivity 10 that are not shown by the phases individually. This rationale has been applied to ionic and mixed electronic/ionic conductors ('heterogeneous doping') ${ }^{11-13}$, where this powerful technique, relying on the introduction of space-charge zones, has been proven to be on a par, if not superior, to the classic homogeneous (bulk) doping (see Supplementary Note 1). While superconducting electronic accumulation layers have been created at heterojunctions between two phases or at grain boundaries (for example refs 8-10, 14), the insertion of an atomic plane of defined interfacial charge (here substitutional insertion) yielding local high-temperature superconductivity has not been achieved so far. This is not only exciting in terms of generating space-dependent superconductivity without dopant disorder but also for purposeful functional structuring.

As well known, homogeneously doped $\mathrm{La}_{2}-\mathrm{Sr}_{x} \mathrm{CuO}_{4}$, in which the resulting strontium (Sr) dopants $\left(\mathrm{Sr}_{\mathrm{La}}^{\prime}\right.$ in Kröger-Vink notation) give rise to a significant concentration of electron holes [h'], exhibits high-temperature superconductivity with critical temperatures $\left(T_{\mathrm{c}}\right)$ up to $\approx 40 \mathrm{~K}$ (for the optimal doping $x=0.16$ ). For a higher doping level ('overdoping'), $T_{c}$ decreases and the structure eventually becomes metallic ${ }^{15,16}$. This is attributed to an interplay of electronic and structural reasons, since for a high Sr-content defect chemistry predicts a tendency to oxygen vacancy $\left(\mathrm{V}_{\mathrm{O}}^{\bullet \bullet}\right)$ compensation (because of double-positive charge), association between $\mathrm{Sr}_{\mathrm{La}}^{\prime}$ and $\mathrm{V}_{\mathrm{O}}^{\bullet \bullet}$ and between $\mathrm{Sr}_{\mathrm{La}}^{\prime}$ and $\mathrm{h}^{\bullet}$ (refs 17,18; see Supplementary Fig. 1 and Supplementary Note 2).

Unlike forming $\mathrm{La}_{2}{ }_{x} \mathrm{Sr}_{x} \mathrm{CuO}_{4}$ solid solutions, we use here unconventional heterogeneous doping ${ }^{11-13}$ to enhance the hole concentration and thus trigger superconductivity. Inspired by the so-called delta-doping technique ${ }^{19-21}$, we replace single $\mathrm{LaO}$ planes with $\mathrm{SrO}$ ones, with the aim of creating two-dimensional (2D) and atomically confined charged layers within the undoped $\mathrm{La}_{2} \mathrm{CuO}_{4}$ matrix. So far, delta-doping in oxides referred to either the insertion of one (or more) complete unit cells of a doped material in a matrix of the same but undoped material ${ }^{22-24}$ or, in few cases dealing with the formation of $2 \mathrm{D}$ electronic gases or local modification of magnetic properties, the successful substitution of single atomic planes in perovskites $(\mathrm{LaO}$ for $\mathrm{SrO}$ planes $^{5,25-27}$, SrO for GdO planes ${ }^{28}$ ). None of them used deltadoping for obtaining high-temperature superconductivity. Either comparatively thick layers were inserted ${ }^{9,29}$ or, in the few cases where $2 \mathrm{D}$ doping was employed ${ }^{5,25-28}$, superconductivity was not achieved.

Here the Sr-to-La substitution targets a single atomic layer of $\mathrm{La}_{2} \mathrm{CuO}_{4}$ and yields a layer of high effective negative charge density. Such an approach is expected to yield a local spacecharge redistribution of $\mathrm{h}^{\bullet}$ and mobile ionic charges ${ }^{12}$. While the possibility of cation redistribution deserves a closer inspection (see below), oxygen diffusion kinetics ${ }^{18}$ (Supplementary Fig. 2) certainly allow oxygen interstitials $\left(\mathrm{O}^{\prime \prime}{ }_{\mathrm{i}}\right)$ and vacancies $\left(\mathrm{V}_{\mathrm{O}}^{\bullet \bullet}\right)$ to redistribute on the nm scale.

The rationale of this contribution is depicted in Fig. 1, which compares the two cases of homogeneously (zero-dimensional) doped with heterogeneously (2D) doped $\mathrm{La}_{2} \mathrm{CuO}_{4}$. While, in the first case (Fig. 1a,c), at equilibrium all positive (negative) defects are increased (depressed) on Sr-doping in correspondence to the randomly distributed $\mathrm{Sr}$ point defects, in the second case (Fig. 1b,d) the interfacial charge dictates the charge-wise similar but spatially very different behaviour in the space-charge zones. A SrO layer substituting one $\mathrm{LaO}$ layer in the $\mathrm{La}_{2} \mathrm{CuO}_{4}$ cell acts as a negatively charged interface, analogously to a charged grainboundary case in a bicrystal ${ }^{11}$, yet with a priori known interfacial
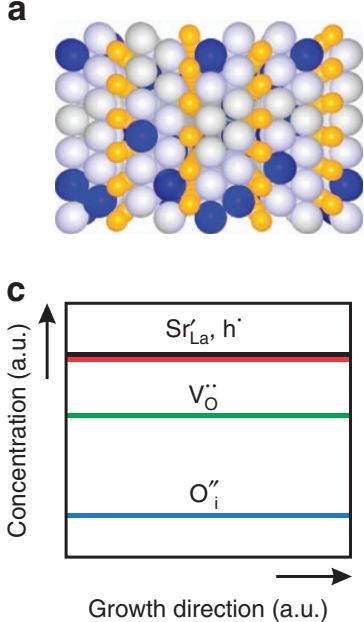

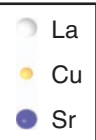

b
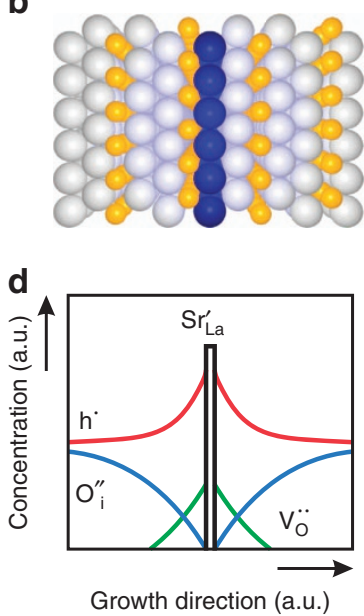

Figure 1 | Homogeneous versus heterogeneous doping for lanthanum cuprate. The crystal structures for two cases are schematically represented in $\mathbf{a}, \mathbf{b}$, respectively (the oxygen ions are omitted for clarity). In $\mathbf{a}, \mathrm{Sr}^{\prime}{ }_{\mathrm{La}}$ are randomly distributed as point defects, whereas in $\mathbf{b}$ they are ordered into a crystallographic plane forming a negatively charged layer. In both cases, in proximity of the $\mathrm{Sr}^{\prime}{ }_{\text {La }}$ defects, structural as well as compositional (that is, formation of compensating charge defects) modifications are expected. The resulting defect concentration profiles are depicted in $\mathbf{c}, \mathbf{d}$ for the zerodimensional and the two-dimensional doping case, respectively. The disordered spatial distribution of $\mathrm{Sr}^{\prime}$ La defects leads, in the first, to a homogeneous increase in hole $\left(h^{\bullet}\right)$ and oxygen vacancy $\left(V_{0}^{\bullet}\right)$ concentration, and to a depressed negatively charged oxygen interstitial $\left(\mathrm{O}^{\prime \prime}{ }_{\mathrm{i}}\right)$ concentration with respect to the undoped material. In $\mathbf{d}$, as a consequence of two-dimensional doping, the formation of a sharp hole accumulation layer with the space-charge layer is predicted.

charge, resulting in an enhancement of the positively charged mobile defects (particularly holes) in the adjacent layers.

In the classical doping case, the probability of finding the excess hole in the local environment of a Sr-dopant is enhanced; yet, owing to the disorder, a high concentration of Cooper pairs in a given layer cannot build up. The transition to an ordered $2 \mathrm{D}$ situation corresponds to a transition from a Debye-Hückel to a Gouy-Chapman situation, in which the very high local interfacial charge, whose sign is known a priori, produces a sharp hole accumulation enabling measurability (see below), percolation efficiency and structurability.

Qualitatively, the effects of homogeneous and heterogeneous doping can be described by the following rules ${ }^{30}$ :

$$
\frac{z_{\mathrm{k}} \delta c_{\mathrm{k}}}{\delta q_{\mathrm{dop}}}<0
$$

where $c_{\mathrm{k}}$ and $z_{\mathrm{k}}$ are the concentration of the charge carrier $k$ and its charge number, respectively, and $q_{\text {dop }}$ the charge of the dopant. This expression indicates that on homogeneous addition of acceptors into a host material (for strontium substituting lanthanum $\left.q_{\text {dop }}<0\right)$ ) the concentrations of all positively (negatively) charged carriers $c_{\mathrm{k}}$ are increased (decreased), as long as they are mobile and can attain distribution equilibrium. This follows from charge conservation and the electroneutrality 
of the disorder reactions. In the case of heterogeneous doping :

$$
\frac{z_{\mathrm{k}} \delta c_{\mathrm{k}}}{\delta \Sigma_{\text {int }}}<0,
$$

where $\sum_{\text {int }}$ represents the effective charge density of the $2 \mathrm{D}$ dopant. Equation (2) follows from the fact that all charge carriers perceive the same electrical potential ${ }^{30}$.

By employing atomic-layer-by-layer oxide molecular beam epitaxy technique (ALL-oxide MBE), which allows for a definition of the nominal composition of each atomic layer ${ }^{31,32}$, we synthesized more than 100 epitaxial superlattices, in which single $\mathrm{LaO}$ layers in the $\mathrm{La}_{2} \mathrm{CuO}_{4}$ structure were substituted by SrO layers with the desired periodicity (see Supplementary Methods and Supplementary Figs 3-5 for preparation and structural characterization). Ignoring oxygen defects, the resulting composition of the heterostructures is described as (Fig. 2a):

$$
\left[\mathrm{SrO}-\mathrm{LaO}-\mathrm{CuO}_{2}+N \times\left(\mathrm{LaO}-\mathrm{LaO}-\mathrm{CuO}_{2}\right)\right] \times R,
$$

with $R$ representing the number of superlattice unit repetitions and $N$ the spacing between the SrO layers (expressed in number of constituting $\mathrm{La}_{2} \mathrm{CuO}_{4}$ units, which corresponds to half a unit cell).

The two sides of each SrO plane (the downward side facing the substrate and the upward side oriented towards the free surface) exhibit remarkably different properties. At the downward side, a clear decoupling between hole and $\mathrm{Sr}$ concentration, whose profile is here abrupt, is detected. This finding proves spacecharge effect as the key mechanism yielding superconductivity. At the upward side instead, where Sr redistribution occurs, superconductivity arises because of conventional homogeneous doping.

\section{Results}

Resistivity measurements reveal confined superconductivity. The in-plane resistivity data collected from a set of samples having comparable total thicknesses $(\approx 400 \AA)$ and different spacing $N$ between subsequent $\mathrm{SrO}$ layers is shown in Fig. $2 \mathrm{~b}$. Notably, the samples exhibit high- $T_{\mathrm{c}}$ superconductivity, with $T_{\mathrm{c}}$ (Fig. 2c) reaching a maximum value of $\sim 35 \mathrm{~K}$ for $5 \leq N \leq 9$ (see Supplementary Methods as well as Supplementary Figs 6 and 7 for the definition and analysis of $T_{\mathrm{c}}$ ). For higher spacing $N, T_{\mathrm{c}}$ saturates at only $\approx 25 \mathrm{~K}$ and the in-plane resistivity drastically increases. This lowering of the $T_{c}$ for large $N$ is connected with a lower orthorhombicity ${ }^{32}$, a trend that is observed also in the case of conventional doping. These results suggest that conducting layers are formed in the proximity of each SrO layer. For short distances between two subsequent SrO layers (namely for $N<5$ ), $T_{\mathrm{c}}$ and resistivity decrease, suggesting 'overdoping' or structural distortions. For $N \leq 1$, a non-superconducting phase is formed.

Abrupt Sr profile at the downward side of the interface. To elucidate the local microstructure and composition, high-resolution a

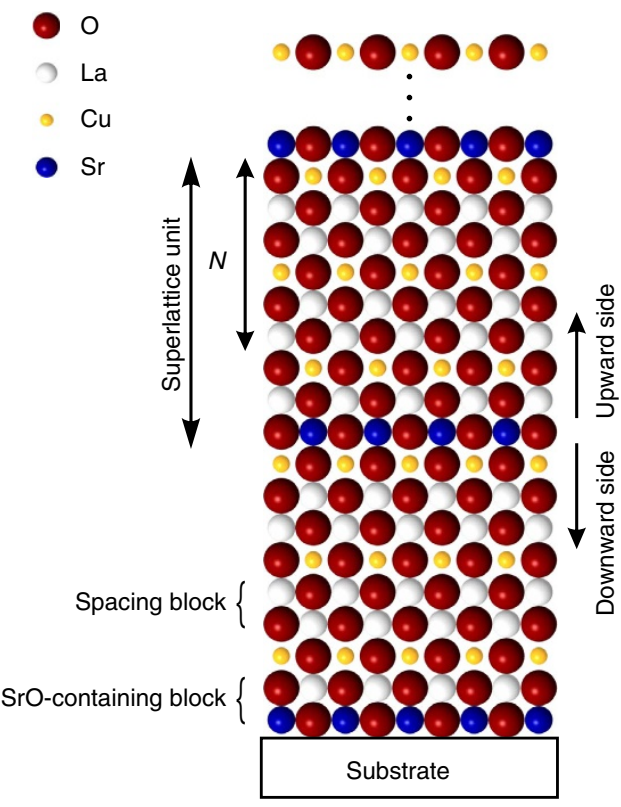

b

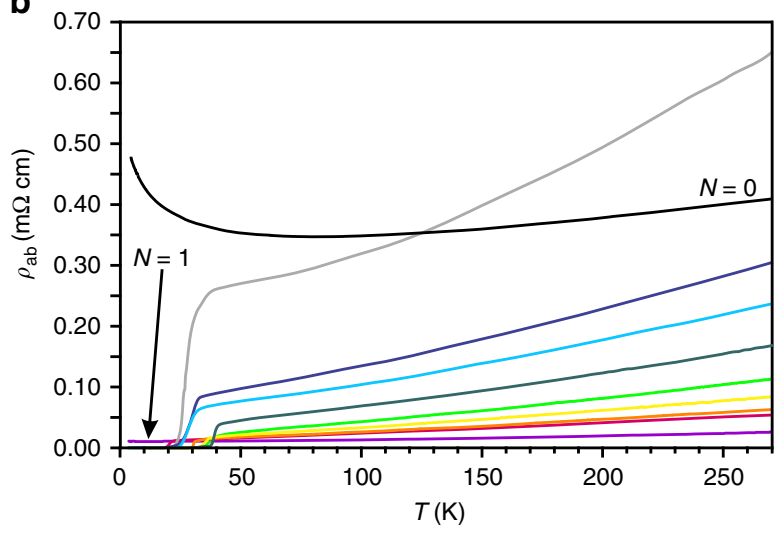

C

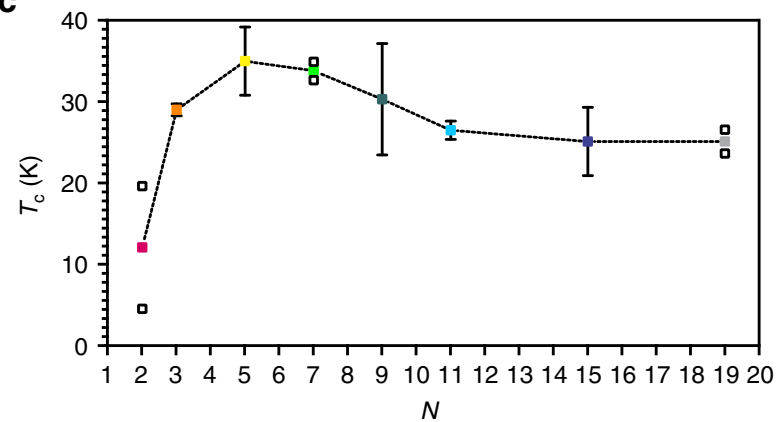

Figure 2 | Electrical properties of two-dimensionally doped $\mathbf{L a}_{\mathbf{2}} \mathbf{C u O}_{\mathbf{4}}$ superlattices. In $\mathbf{a}$, a sketch of the structure is represented. Starting from the $\mathrm{La}_{2} \mathrm{CuO}_{4}$ structure, superlattices are formed by regularly replacing single layers of $\mathrm{LaO}$ with $\mathrm{SrO}$ planes. The general formula can be written as: [SrO-LaO$\left.\mathrm{CuO}_{2}+\mathrm{N} \times\left(\mathrm{LaO}-\mathrm{LaO}-\mathrm{CuO}_{2}\right)\right] \times R$. The periodicity $\mathrm{N}$ is defined as the number of spacing blocks between two SrO-containing blocks, whereas $R$ indicates the number of superlattice units. (b) Representative in-plane resistivity measurements for superlattices with different spacing $N$, epitaxially grown along the c-direction on $\mathrm{LaSrAlO}_{4}$ (001) substrates. Each spacing is marked with a different colour as defined in c, in which the solid square symbols represent the value of the critical temperature $T_{c}$ plotted as a function of $N$. Here the error bars show the s.d. of the $T_{c}$ values obtained for a set of samples having the same composition. For those compositions (value of $N$ ), in which a set of two samples was considered for the determination of the average critical temperature, both $T_{\mathrm{c}}$ data points are shown in the diagram (open square symbols). The uncertainty in the definition of the $T_{\mathrm{c}}$ value can be ascribed to normal sample-to-sample variations due to, for example, light stoichiometry offset, substrate roughness and strain. 
transmission electron microscopy, together with analytical characterizations, was systematically performed employing aberrationcorrected scanning transmission electron microscopy (TEM, see also Supplementary Methods). The high-angle annular dark-field (HAADF) micrograph in Fig. 3a reveals that the superlattice exhibits perfect epitaxy, illustrating the absence of any extended structural defects such as misfit dislocations or antiphase boundaries. A high-resolution micrograph is presented in Fig. 3e, showing the atomic arrangement in greater detail. In the intensity profile obtained from the HAADF image (averaged perpendicularly to the growth direction), the intensity drop is connected with the Sr-containing layers (compare Fig. 3a,b). It is noteworthy that, such a change of intensity involves more than a single atomic plane indicating a certain $\mathrm{Sr}$ redistribution into the $\mathrm{La}_{2} \mathrm{CuO}_{4}$ matrix. Highly spatial-resolved spectroscopic analyses (Fig. $3 \mathrm{c}, \mathrm{d}$ ) provide more precise information revealing a pronouncedly asymmetric character of the Sr profile: virtually abrupt at the side facing the substrate (downward side) with an extent of $0.9 \pm 0.2 \mathrm{~nm}$ and smeared over $2.3 \pm 0.4 \mathrm{~nm}$ at the upward side, a feature that will be explained below. Results from energy-dispersive X-ray spectroscopy (EDXS) allows for a quantitative estimation of the $\mathrm{La}^{3+}$ / $\mathrm{Sr}^{2+}$ concentration ratio (Fig. 3c and Supplementary Fig. 8). The maximum estimated ratio, obtained by averaging the maximum intensities of the different peaks, is $0.21 \pm 0.02$. Electron energy-loss spectroscopy (EELS; Fig. 3d) provides further robust evidence of the asymmetric Sr distribution, ensuring single atomic layer resolution (step size $\approx 2 \AA)^{33,34}$.

By averaging the different EELS Sr- $\mathrm{L}_{2,3}$ intensity profiles from several Sr-containing atomic slabs, and applying an appropriate scaling factor to satisfy the nominal overall composition of the compound, one can accurately define the Sr level $(x)$ that can be assigned to each 'constituting block' (namely a single $\mathrm{CuO}_{2}$ plane and the two surrounding ( $\mathrm{La}, \mathrm{Sr}) \mathrm{O}$ layers) in proximity of the layer where $\mathrm{Sr}$ was initially inserted (see Fig. 3f and also Supplementary Methods, Supplementary Figs 9 and 10). Obviously, we have realized a rather abrupt profile, but only at the downward side, while at the other side (upward) there is a pronounced redistribution of the aliovalent cation. According to our rationale, we expect the space-charge mode to be active at the downward side and a usual homogeneous mode at the upward side ( $c f$. Supplementary Note 3 and Supplementary Fig. 11). Taking both these mechanisms into account, the expected hole profile is sketched in Fig. 4.

Zn tomography specifies layer-dependent superconductivity. Further complementary tests relying on the $\mathrm{Zn}$-doping tomography technique ${ }^{21}$ were carried out on structures containing one single SrO layer (Fig. 5a). The method consists of substituting $\sim 3 \%$ of $\mathrm{Cu}$ with $\mathrm{Zn}$ and thus suppressing $T_{\mathrm{c}}$ of specific $\mathrm{CuO}_{2}$ planes (Supplementary Fig. 12 and Supplementary Methods). In this way, spatial information is available, which allows us to identify which particular $\mathrm{CuO}_{2}$ planes are responsible for high- $T_{\mathrm{c}}$ superconductivity. To determine the contribution to superconductivity of each single $\mathrm{CuO}_{2}$ plane at the downward side of the interface, we proceeded as follows: all the $\mathrm{CuO}_{2}$ planes at the upward side were $\mathrm{Zn}$-doped but on the downward side selected single $\mathrm{CuO}_{2}$ planes were $\mathrm{Zn}$-doped and the electrical transport properties measured. Analogously, the same procedure was applied to determine the contribution of each single $\mathrm{CuO}_{2}$ layer of the upward side, after that the $P=-2 \mathrm{CuO}_{2}$ plane of the downward side had been $\mathrm{Zn}$-doped.

The results are summarized in Fig. $5 b$, in which $T_{c}$ obtained after having placed $\mathrm{Zn}$ in the different $\mathrm{CuO}_{2}$ planes is shown (see also Supplementary Fig. 13). The two sides exhibit similar $T_{\mathrm{c}}$ (orange and green areas in Fig. 5b for downward and upward
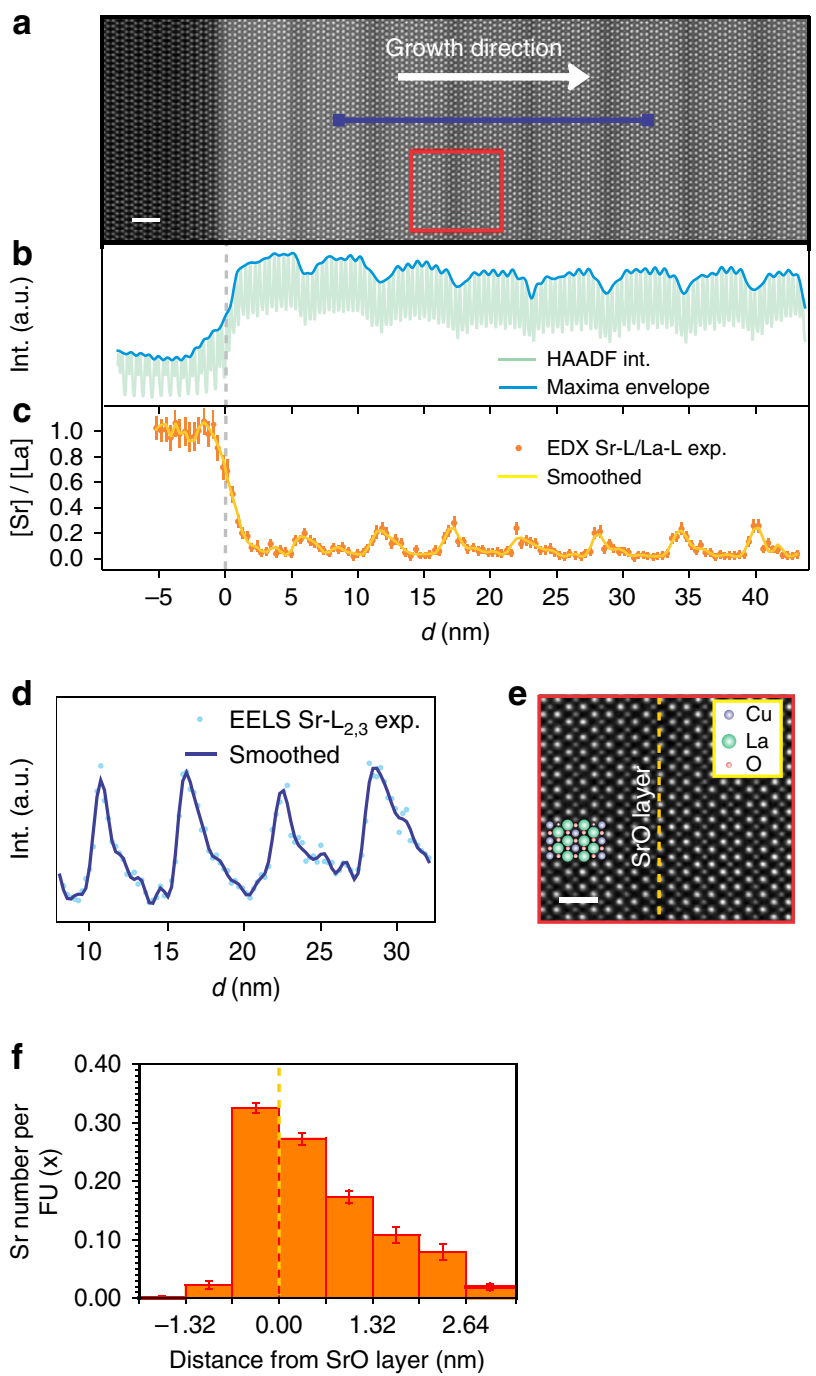

Figure 3 | High-resolution transmission electron microscopy (HRTEM) analysis of cationic distribution in two-dimensional doped $\mathrm{La}_{2} \mathrm{CuO}_{4}$.

(a) HAADF-STEM image of two-dimensionally doped $\mathrm{La}_{2} \mathrm{CuO}_{4}$ showing the microstructure of a superlattice $(R=8, N=7)$ grown on the $\mathrm{LaSrAlO}_{4}$ (001) substrate. The alternation of brighter and darker areas reflects the superlattice structure, in which Sr-doped regions (dark) are separated by undoped $\mathrm{La}_{2} \mathrm{CuO}_{4}$ (bright). This is clearly shown by the maxima envelope of the image-intensity profile, integrated perpendicular to the growth direction (b, dark blue line). Scale bar, $2 \mathrm{~nm}$. In $\mathbf{b}$, the intensity oscillations of the intensity profile due to the different contrast of each atomic layer (green line) can be seen. A magnified image of the region highlighted in red in $\mathbf{a}$ is shown in $\mathbf{e}$, in which the dotted yellow line corresponds to the layer having maximum $\mathrm{Sr}$ content. Scale bar, $1 \mathrm{~nm}$. (c) [Sr]/[La] ratio, extracted from an EDXS line scan across the region shown in a. Asymmetric $\mathrm{Sr}$ distribution, smeared in the growth direction, is detectable. Sr-L and La-L lines were used for quantifying the $\mathrm{Sr}$ concentration, and the integrated signals of $\mathrm{Sr}$ and La were calibrated using the substrate region where $[\mathrm{La}] /[\mathrm{Sr}]$ is equal to unity (see Supplementary Fig. 8 for the net counts of $\mathrm{Sr}-\mathrm{L}$ and La-L EDXS lines). Here error bars are the square root of the intensity. A similar $\mathrm{Sr}$ asymmetric profile results from the integration of the $\mathrm{Sr}_{-} \mathrm{L}_{2,3}$ EELS line profiles, as shown in $\mathbf{d}$, which has been acquired across four Sr-containing layers (blue line in $\mathbf{a}$ ). Here the error bars (square root of the intensity) are smaller than the symbols. From the EELS analysis, the average $\mathrm{Sr}$ number per formula unit FU ( $x$ in $\mathrm{La}_{2}{ }_{-} \mathrm{Sr}_{x} \mathrm{CuO}_{4}$ ), for each ( $\mathrm{La}, \mathrm{Sr}) \mathrm{O}-\mathrm{CuO}_{2}-(\mathrm{La}, \mathrm{Sr}) \mathrm{O}$ 'constituting block' in proximity of the Sr-containing layers, as depicted in $\mathbf{f}$, was obtained (the s.d. is represented by the error bars). 


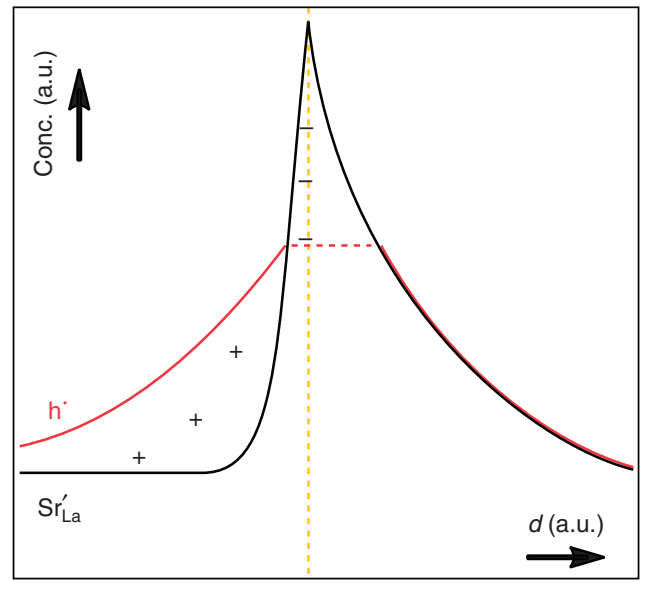

Figure 4 | Expected hole profile in the Sr-doped region. As a consequence of $\mathrm{Sr}$ distribution, the expected hole profile is sketched (see also Supplementary Fig. 11). Hole and Sr spatial distributions are depicted in red and black, respectively. While on the right-hand (upward) side the hole concentration follows the Sr profile (cf. Fig. 1c), on the left-hand (downward) side a space-charge situation occurs, resulting in an accumulation layer profile (cf. Fig. 1d). The dotted yellow line represents the nominal position of the SrO layer.

sides, respectively), in contrast to what one would expect according to a simple homogeneous doping picture (see section above). Moreover, while no remarkable effect coming from $\mathrm{Zn}$ substitution in single $\mathrm{CuO}_{2}$ planes could be observed for the upward side (consistent with a homogeneous doping situation predicting several $\mathrm{CuO}_{2}$ planes to be superconducting, $c f$. Fig. $3 \mathrm{f}$ ), for the downward side, we found $T_{\mathrm{c}}$ to stay almost unaffected when $\mathrm{Zn}$ was put in each plane $P(-4 \leq P \leq 0)$, except for $P=-2$. In this case, the insertion of $\mathrm{Zn}$ leads to a drastic $T_{\mathrm{c}}$ reduction $\left(T_{c}<4 \mathrm{~K}\right)$, allowing us to identify this $\mathrm{CuO}_{2}$ plane $(P=-2)$ as the main source of high- $T_{\mathrm{c}}$ superconductivity of the downward side. Owing to the very low local Sr content $(x \approx 0.02)$, this undoubtedly demonstrates that superconductivity at the downward side does not arise from homogeneous doping. Rather, one has to refer to the heterogeneous case as sketched in Fig. 1d, which results in a hole accumulation, up to the optimal for superconductivity, for $P=-2$. Please note again that, in all the samples, which were prepared to investigate the role of the downward side of the interface by $\mathrm{Zn}$ tomography, all the $P \geq 1$ planes were doped by $\mathrm{Zn}$ with the purpose of suppressing any contribution to $T_{\mathrm{c}}$ stemming from the upward side.

Electrical properties of the $\mathrm{La}_{2} \mathrm{CuO}_{4} / \mathrm{SrO}$ bilayer. To confirm the minor role of the $\mathrm{La}^{3+} / \mathrm{Sr}^{2}+$ intermixing in the definition of the electrical properties at the downward side, we also considered a structure consisting of a bilayer of $\mathrm{La}_{2} \mathrm{CuO}_{4}$ and a 10 -unit-cellthick SrO film (Fig. 6a). Here the growth dynamics for the downward side follows a similar sequence compared with the structure depicted in Fig. 5a, with the only difference that several SrO layers are deposited in succession. If cation intermixing was responsible for the observed superconductivity at the backward side of the SrO planes, then we would expect to detect superconductivity also in this bilayer as long as we can neglect serious accommodation problems of the replaced La. Nevertheless, no sign of metallicity or superconductivity could be found (Fig. 6b, Supplementary Note 4 and Supplementary Fig. 14), confirming the relevance space-charge effects induced by the heterogeneous doping mechanism.

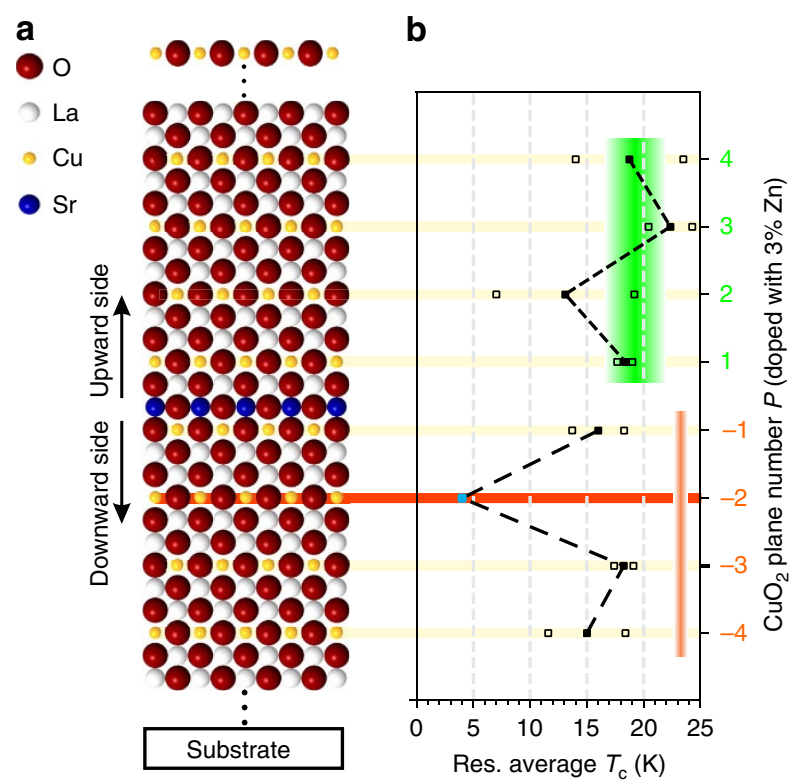

Figure 5 | Zn tomography on two-dimensionally doped $\mathrm{La}_{2} \mathrm{CuO}_{4}$. (a) Sketch of the symmetric structure investigated by $\mathrm{Zn}$ tomography (the $\mathrm{SrO}$ atoms are depicted in blue). By means of $\mathrm{Zn}$-doping, one can suppress the superconducting critical temperature of selected $\mathrm{CuO}_{2}$ planes in proximity of the SrO layer, thus mapping $T_{c}$ with atomic layer precision. The results are shown in $\mathbf{b}$, where the average residual $T_{\mathrm{c}}$ (closed squares) is plotted as a function of the $\mathrm{Zn}$-doped $\mathrm{CuO}_{2}$ plane $P$. One can see that doping by $Z n$ the $P=-2$ plane (marked in red) leads to a substantial reduction of $T_{\mathrm{c}}$, indicating that this plane is mainly responsible for superconductivity at the downward side. The indicated $T_{\mathrm{c}}$ value (closed light blue square) has to be considered as an upper limit, since the critical temperature for the samples was below the low limit for our measurements $(4 \mathrm{~K})$. As for the upward side, doping by $\mathrm{Zn}$ single $\mathrm{CuO}_{2}$ planes does not have a pronounced effect, indicating that several planes are responsible for $T_{\mathrm{c}}$. Reference $T_{\mathrm{c}}$ for the upward and downward sides are indicated in green and orange, respectively. Open squares indicate the single data points.

STEM-EELS confirms the predicted hole profile. A further nice corroboration of the findings described above stems from the intensity profile of the oxygen-K-edge pre-peak ${ }^{35}$, which is attributed to electronic transitions from the $\mathrm{O}_{1 \mathrm{~s}}$-core level to hole states with $p$ symmetry in the valence band ( $c f$. Supplementary Fig. 15). As these data are averaged over each ( $\mathrm{La}, \mathrm{Sr}) \mathrm{O}-\mathrm{CuO}_{2}-$ (La,Sr)O-constituting block, we compare it with the averaged $\mathrm{Sr}$ profile obtained from EELS analysis in Fig. 3f. The scaling was performed to satisfy global electroneutrality (ignoring oxygen point defects-see Supplementary Methods). The results are shown in Fig. 7. One clearly recognizes local electroneutrality on the right-hand side (upward side) and space-charge accumulation on the left-hand side (downward side). The qualitative agreement with the prediction given in Fig. 4 is remarkable. In addition, the absolute value of the hole concentration $\left[\mathrm{h}^{\bullet}\right]$ is reasonable: to plane $P=-2$ corresponds a hole concentration $\approx 0.1$ per $\mathrm{CuO}_{2}$ plane, which for single phase thin films on $\mathrm{LaSrAlO}_{4}(001)$ substrates has $T_{\mathrm{c}} \approx 25 \mathrm{~K}$ (refs 15,21), as indeed estimated by the $\mathrm{Zn}$-doping experiment ( $c f$. Fig. 5b). This agreement in absolute numbers may be partially coincidental because of possible structure distortions ${ }^{36}$, and to the fact that the high- $T_{\mathrm{c}}$ behaviour in space-charge zones may differ from the bulk situation. 


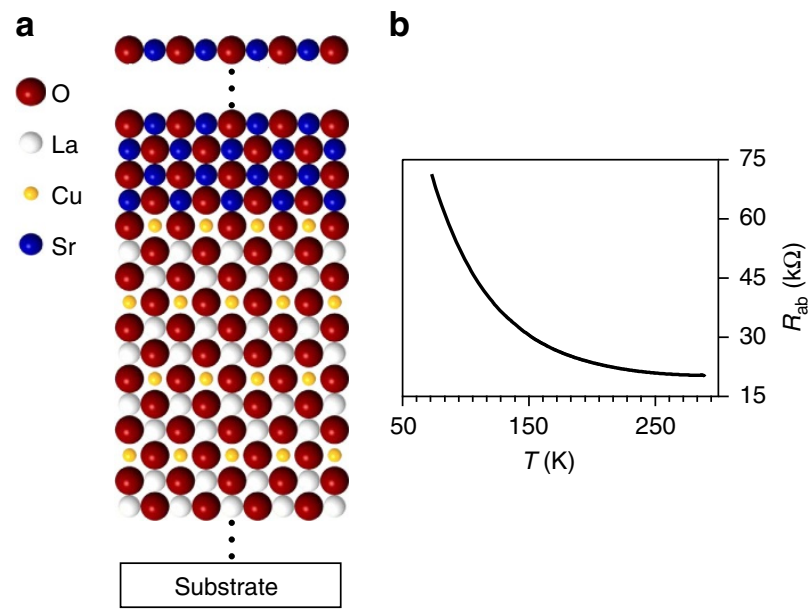

Figure 6 | Electrical behaviour of the $\mathrm{La}_{2} \mathrm{CuO}_{4} / \mathrm{SrO}$ bilayer. (a) Sketch of the $\mathrm{La}_{2} \mathrm{CuO}_{4} / \mathrm{SrO}$ bilayer structure. Unlike the structure represented in Fig. $5 \mathrm{a}$, where the $\mathrm{SrO}$ is an effective atomic layer in the $\mathrm{La}_{2} \mathrm{CuO}_{4}$ structure, thus being negatively charged with respect to the replaced $\mathrm{LaO}$ layer, here it forms a neutral second phase (a thick $\mathrm{SrO}$ layer). The electrical properties of such a structure ( 15 u.c. (unit cell) $\mathrm{La}_{2} \mathrm{CuO}_{4} / 10$ u.c. SrO), exhibiting semiconducting behaviour, are illustrated in $\mathbf{b}$

\section{Discussion}

The most fascinating of the above described results regards the realization of a space-dependent superconductivity induced, as a consequence of a space-charge effect, by the $\mathrm{Sr}$ abrupt profile at the downward side of the SrO plane. While EDXS and EELS $\mathrm{Sr}-\mathrm{L}_{2,3}$ intensity profiles reveal the $\mathrm{Sr}$ concentration profile to abruptly decrease even in close proximity of the $\mathrm{SrO}$ plane, the analysis of the pre-peak of the oxygen-K-edge indicates a substantial accumulation of $\mathbf{h}^{\bullet}$ within the same region. Electrical transport measurements carried out according to the $\mathrm{Zn}$ tomography technique corroborate these findings by demonstrating that the $\mathrm{CuO}_{2}$ plane-labelled $P=-2$ is the main source of high- $T_{\mathrm{c}}$ superconductivity at the downward side. Such hole accumulation (decoupled from $\mathrm{Sr}$ ), due to the excess negative charge of the SrO layer, leads to a highly space-dependent $T_{\mathrm{c}}$. The decay length of the hole profile is consistent with the bulk defect chemistry of pure $\mathrm{La}_{2} \mathrm{CuO}_{4}$ governed by $2\left[\mathrm{O}^{\prime \prime}{ }_{\mathrm{i}}\right]=\left[\mathrm{h}^{\bullet}\right]^{17}$.

It is noteworthy that, while in the case of homogeneous doping a rather large volume is necessary for inducing percolating superconductivity ${ }^{22,37}$, a space-charge-induced two-dimensional layer serves for highest volume concentration in terms of Cooper pairs. (As to the influence of dimensionality on superconductivity cf. ref. 38). Moreover, heterogeneous doping offers the advantage of experimentally probing the space-charge-induced superconductivity by means of different and complementary techniques, including STEM-EELS (scanning transmission electron microscopy-EELS) and Zn-doping tomography. In the literature, there is only one report on a possible decoupling between $h^{\bullet}$ and $\mathrm{Sr}_{\mathrm{La}}^{\prime}$ at the interface between metallic $\mathrm{La}_{2-x} \mathrm{Sr}_{x} \mathrm{CuO}_{4}$ and insulating $\mathrm{La}_{2} \mathrm{CuO}_{4}$ (ref. 10), yet also at the upward side (in growth direction) of the interface where we rather observe Sr redistribution.

At this side, the situation is dominated by non-negligible dopant redistribution leading to $h^{\bullet}$ locally neutralizing diffused $\mathrm{Sr}_{\mathrm{La}}^{\prime}$ point defects. Such mixing effects have been seen in MBEgrown $\delta$-doped semiconductors ${ }^{19,39}$ and, in some cases, in PLDgrown complex oxide systems ${ }^{40,41}$. Its occurrence is due to the specific kinetics of the atomic-layer-by-layer deposition process (Supplementary Methods), assuming intermixing to occur only

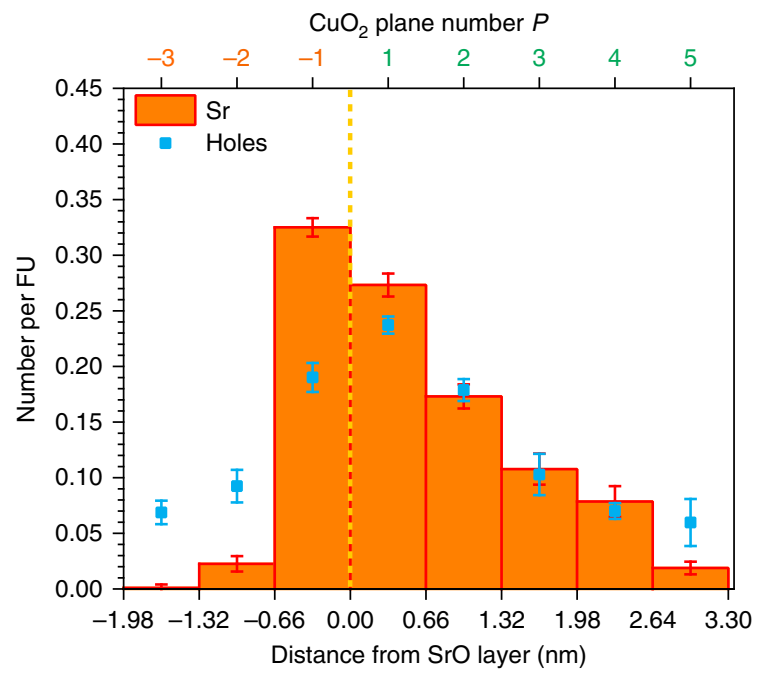

Figure 7 | Comparison of Sr and hole concentration profiles as obtained by STEM-EELS. The number of holes per formula unit assigned to each constituting block ( $\mathrm{La}, \mathrm{Sr}) \mathrm{O}-\mathrm{CuO}_{2}-(\mathrm{La}, \mathrm{Sr}) \mathrm{O}$ for a structure having spacing $N=7$, as obtained by the O-K-edge EELS analysis (see Supplementary Fig. 10 and Supplementary Methods-s.d. indicated by error bars) is reported here in comparison with the Sr profile as depicted in Fig. 3f. The $\mathrm{CuO}_{2}$ plane numbering is made in agreement with Fig. 5. Note the decoupling between the two profiles at the downward side interface, and the far-reaching agreement with Fig. 4.

between the last atomic layers on the film surface and the incoming species of high-kinetic energy. This may also be connected with the observed roughness of the film (Supplementary Fig. 16) and the high surface atomic mobility during growth. In such a nonequilibrium situation, cation exchange occurs in the growth direction only ${ }^{42}$, whereas it is limited in the downward direction because of sluggish diffusion. Finally, dopant segregation to the surface of the heterostructure ${ }^{43}$, which has been theoretically predicted for $\mathrm{La}_{2} \mathrm{CuO}_{4}$ (ref. 44), provides an additional, mainly energetic, driving force for the formation of such a $\mathrm{Sr}$ profile.

In summary, this study demonstrates the significance of heterogeneous doping in the field of superconductivity. It represents not simply an alternative to the homogenous doping approach but rather it offers a series of unique features. First, in the case of 2D doping, one can tune the local charge distribution (and thus local superconductivity) without affecting the chemical composition of the host material and without introducing local crystallographic disorder resulting from the dopant solid solution. This allows for a clear space-charge effect and for structuring the matrix material at wish, that is, we are able to insert active layers in the matrix and control their number, position and relative distance. From an operational point of view, it is certainly desired and will be attempted in the future to arrive at a two-sided abruptness of such profiles rather than an asymmetric situation.

Second, one can induce layer-dependent high-temperature superconductivity, while conventional doping leads to a homogeneous situation. Third, such superconductivity induced by a $2 \mathrm{D}$ charge distribution yields the formation of a layer of high Cooper pair concentration unlike the case of induction by homogeneously distributed dopant atoms. Fourth, the technique may be applied to situations where the solubility of the dopant is limited (and conventional doping is not possible) and/or a large volume is required for a sufficient percolation.

Last, our findings can be used to shed light on the influence of dimensionality on superconductivity ${ }^{38}$ but also on the 
importance of typically neglected effects of defect chemistry and ionic mobility in the field of high-temperature superconductivity.

\section{Methods}

ALL-oxide MBE synthesis. Thin films of 2D Sr-doped lanthanum cuprate were grown on $\mathrm{LaSrAlO}_{4}(001)$ substrates (Crystec $\mathrm{GmbH}$ ), using ozone-assisted $\mathrm{MBE}^{31,32}$. The deposition conditions used for synthesizing the samples are temperature $T \approx 620^{\circ} \mathrm{C}$ and pressure $p \approx 2.5 \times 10^{-5}$ Torr (of mixed ozone, molecular and atomic oxygen). It is worth noting that, after the growth, all the samples were cooled under vacuum starting from a temperature $T=200^{\circ} \mathrm{C}$, which ensures that no contribution to electrical conductivity of $\mathrm{La}_{2} \mathrm{CuO}_{4}$ can be ascribed to extrinsic doping by interstitial oxygen ( $c f$. ref. 32). A more detailed description of the growth method and sample structural characterization can be found in the Supplementary Methods.

Conductivity measurements. Electrical properties of the films were probed using direct current (DC)-electrical conductivity measurements as a function of temperature, using a Keithley 2,000 multimeter and a Keithley 2,400 sourcemeter implemented in a home-built system. The measurements were performed by warming up the sample from $\approx 4 \mathrm{~K}$ to room temperature, with a rate $<0.1 \mathrm{~K} \mathrm{~s}^{-1}$ applied by a motorized dipstick whose motion was controlled via the customized LABVIEW software. Pt contacts were deposited on sample corners by sputtering, and measurements were carried out in a four-point (Van Der Pauw) configuration. To minimize any contribution due to possible sample inhomogeneities, several measurements were carried out on the same sample on rotation.

Electron microscopy and spectroscopy. The cross-section specimens for STEM experiments were thinned to electron transparency by tripod polishing down to $\approx 10 \mu \mathrm{m}$ and argon ion beam milling in a stage cooled with liquid nitrogen. The electron microscopy and spectroscopy measurement were performed on a JEOL ARM 200CF microscope equipped with a cold field-emission electron source, a probe $\mathrm{C}_{\mathrm{s}}$ corrector, a large solid-angle SDD-type JEOL Centurio EDX detector and a Gatan GIF Quantum ERS spectrometer. The microscope was operated at $200 \mathrm{kV}$, a semiconvergence angle $(\alpha)$ of $21 \mathrm{mrad}$, giving rise to a probe size of $0.8 \AA$ ( $1 \AA$ for the analytical analysis). Collection angle (109-270 mrad) was used to obtain the HAADF images. A collection semi-angle $(\beta)$ of $68.5 \mathrm{mrad}$ was used for EELS measurements. The STEM analysis was carried out at the specimen region where thickness is below $30 \mathrm{~nm}(t / \lambda<0.4$ measured by low loss EELS using a log-ratio method). A detailed analysis about EELS data evaluation can be found in the Supplementary Methods and Supplementary Figs 9 and 10.

\section{References}

1. Takahashi, K. S., Kawasaki, M. \& Tokura, Y. Interface ferromagnetism in oxide superlattices of $\mathrm{CaMnO}_{3} / \mathrm{CaRuO}_{3}$. Appl. Phys. Lett. 79, 1324-1326 (2001).

2. Brinkman, A. et al. Magnetic effects at the interface between non-magnetic oxides. Nat. Mater. 6, 493-496 (2007)

3. Gabay, M. \& Triscone, J.-M. Hund rules with a twist. Nat. Phys. 9, 610-611 (2013).

4. Ruhman, J., Joshua, A., Ilani, S. \& Altman, E. Competition between Kondo screening and magnetism at the $\mathrm{LaAlO}_{3} / \mathrm{SrTiO}_{3}$ interface. Phys. Rev. B 90, 125123 (2014)

5. Ohtomo, A., Muller, D.A., Grazul, J.L. \& Hwang, H.Y. Artificial chargemodulation in atomic-scale perovskite titanate superlattices. Nature 419, 378-380 (2002).

6. Ohtomo, A. \& Hwang, H. Y. A high-mobility electron gas at the $\mathrm{LaAlO}_{3} /$ $\mathrm{SrTiO}_{3}$ heterointerface. Nature 427, 423-426 (2004).

7. Hotta, Y., Susaki, T. \& Hwang, H.Y. Polar discontinuity of the $\mathrm{LaVO}_{3} / \mathrm{SrTiO}_{3}$ interface. Phys. Rev. Lett. 99, 236805 (2007).

8. Reyren, N. et al. Superconducting interfaces between insulating oxides. Science 317, 1196-1199 (2007).

9. Biscaras, J. et al. Two dimensional superconductivity at a Mott insulator/band insulator interface $\mathrm{LaTiO}_{3} / \mathrm{SrTiO}_{3}$. Nat. Commun. 1, 89 (2010).

10. Gozar, A. et al. High-temperature interface superconductivity between metallic and insulating copper oxides. Nature 455, 782-785 (2008).

11. Maier, J. Ionic conduction in space charge regions. Prog. Solid State Chem. 23, 171-263 (1995).

12. Maier, J. Nanoionics: ion transport and electrochemical storage in confined systems. Nat. Mater. 4, 805-815 (2005).

13. Lupetin, P., Gregori, G. \& Maier, J. Mesoscopic charge carriers chemistry in nanocrystalline $\mathrm{SrTiO}_{3}$. Angew. Chem. Int. Ed. 49, 10123-10126 (2010).

14. Hammer, G. et al. Enhanced supercurrent density in polycrystalline $\mathrm{YBa}_{2} \mathrm{Cu}_{3} \mathrm{O}_{7-\delta}$ at $77 \mathrm{~K}$ from calcium doping of grain boundaries.. Nature 407, 162-164 (2000)

15. Torrance, J. B. et al. Anomalous disappearance of high- $T_{\mathrm{c}}$ superconductivity at high hole concentration in metallic $\mathrm{La}_{2-\mathrm{x}} \mathrm{Sr}_{\mathrm{x}} \mathrm{CuO}_{4}$. Phys. Rev. Lett. 61, 1127-1130 (1988)
16. Sato, H., Tsukada, A., Naito, M. \& Matsuda, A. $\mathrm{La}_{2-x} \mathrm{Sr}_{x} \mathrm{CuO}_{y}$ epitaxial thin films ( $\mathrm{x}=0$ to 2): Structure, strain, and superconductivity. Phys. Rev. B 61, 12447-12456 (2000).

17. Maier, J. \& Pfundtner, G. Defect chemistry of the high- $T_{\mathrm{c}}$ superconductors Adv. Mater. 3, 292-297 (1991).

18. Opila, E. J., Tuller, H. L., Wuensch, B. J. \& Maier, J. Oxygen tracer diffusion in $\mathrm{La}_{2-x} \mathrm{Sr}_{x} \mathrm{CuO}_{4-y}$ single crystals. J. Am. Ceram. Soc. 76, 2363-2369 (1993).

19. Schubert, E. F. Delta doping of III-V compound semiconductors: Fundamentals and device applications. J.Vac. Sci. Technol. A 8, 2980-2996 (1990).

20. Dingle, R., Stoermer, H.L., Gossard, A.C. \& Wiegmann, W. Electron mobilities in modulation-doped semiconductor heterojunction superlattices. Appl. Phys. Lett. 33, 665-667 (1978).

21. Logvenov, G., Gozar, A. \& Bozovic, I. High-temperature superconductivity in a single copper-oxygen plane. Science 326, 699-702 (2009).

22. Bozovic, I. et al. No mixing of superconductivity and antiferromagnetism in a high-temperature superconductor. Nature 422, 873-875 (2003)

23. Kozuka, Y. et al. Two-dimensional normal-state quantum oscillations in a superconducting heterostructure. Nature 462, 487-490 (2009).

24. Jalan, B., Stemmer, S., Mack, S. \& Allen, S. J. Two-dimensional electron gas in $\delta$-doped $\mathrm{SrTiO}_{3}$. Phys. Rev. B 82, 081103 (2010).

25. Kim, J. S. et al. Nonlinear Hall effect and multichannel conduction in $\mathrm{LaTiO}_{3} /$ $\mathrm{SrTiO}_{3}$ superlattices. Phys. Rev. B 82, 201407 (2010).

26. Santos, T. S. et al. Delta doping of ferromagnetism in antiferromagnetic manganite superlattices. Phys. Rev. Lett. 107, 167202 (2011).

27. Nishio, K. et al. Delta-doped epitaxial $\mathrm{La} \mathrm{SrTiO}_{3}$ field-effect transistor. Appl Phys. Lett. 98, 242113 (2011).

28. Ouellette, D. G. et al. High-density two-dimensional small polaron gas in a delta-doped mott insulator. Sci. Rep. 3, 3284 (2013).

29. Biscaras, J. et al. Multiple quantum criticality in a two-dimensional superconductor. Nat. Mater. 12, 542-548 (2013).

30. Maier, J. in Modern Aspects of Electrochemistry N. 38 (eds Conway, B. E., Vayenas, C. G., White, R. E. \& Gamboa-Adelco, M. E.) 29-60 (Kluwer Academic/Plenum Publisher, 2005).

31. Baiutti, F., Christiani, G. \& Logvenov, G. Towards precise defect control in layered oxide structures by using oxide molecular beam epitaxy. Beilstein J. Nanotechnol. 5, 596-602 (2014).

32. Baiutti, F. Heterogeneous Doping And Superconductivity In $\mathrm{La}_{2} \mathrm{CuO}_{4}-$ Based Heterostructures. Phd thesis (Univ. Stuttgart, 2015).

33. Muller, D. A. et al. Atomic-scale chemical imaging of composition and bonding by aberration-corrected microscopy. Science 319, 1073-1076 (2008).

34. Pennycook, S. J., Varela, M., Lupini, A. R., Oxley, M. P. \& Chisholm, M. F. Atomic-resolution spectroscopic imaging: past, present and future. J. Electron Microsc. 58, 87-97 (2009).

35. Romberg, H., Alexander, M., Nücker, N., Adelmann, P. \& Fink, J. Electronic structure of the system $\mathrm{La}_{2-x} \mathrm{Sr}_{x} \mathrm{CuO}_{4+\delta}$. Phys. Rev. B 42, 8768-8771 (1990).

36. Butko, V. Y., Logvenov, G., Bozovic, N., Radovic, Z. \& Bozovic, I. Madelung strain in cuprate superconductors - a route to enhancement of the critical temperature. Adv. Mater. 21, 3644-3648 (2009).

37. Ruefenacht, A. et al. Growth of single unit-cell superconducting $\mathrm{La}_{2-x} \mathrm{Sr}_{x} \mathrm{CuO}_{4}$ films. Solid State Electron 47, 2167-2170 (2003).

38. Kosterlitz, J.M. \& Thouless, D.J. Ordering, metastability and phase transitions in two-dimensional systems. J. Phys. C 6, 1181-1203 (1973).

39. Muraki, K., Fukatsu, S., Shiraki, Y. \& Ito, R. Surface segregation of In atoms during molecular beam epitaxy and its influence on the energy levels in InGaAs/GaAs quantum wells. Appl. Phys. Lett. 61, 557-559 (1992).

40. Kourkoutis, L. F., Muller, D. A., Hotta, Y. \& Hwang, H. Y. Asymmetric interface profiles in $\mathrm{LaVO}_{3} / \mathrm{SrTiO}_{3}$ heterostructures grown by pulsed laser deposition. Appl. Phys. Lett. 91, 163101 (2007).

41. Kozuka, Y., Hikita, Y., Bell, C. \& Hwang, H. Y. Dramatic mobility enhancements in doped $\mathrm{SrTiO}_{3}$ thin films by defect management. Appl. Phys Lett. 97, 012107 (2010).

42. Pankratov, E. L. Dynamics of $\delta$-dopant redistribution during heterostructure growth. Eur. Phys. J. B 57, 251-256 (2007).

43. Lee, W., Han, J. W., Chen, Y., Cai, Z. \& Yildiz, B. Cation size mismatch and charge interactions drive dopant segregation at the surfaces of manganite perovskites. J. Am. Chem. Soc. 135, 7909-7925 (2013).

44. Kenway, P. R., Parker, S. C. \& Mackrodt, W. C. The calculated structure, stability and composition of the low index surfaces of $\mathrm{La}_{2} \mathrm{CuO}_{4}$ and $\mathrm{Nd}_{2} \mathrm{CuO}_{4}$ Surf. Sci. 326, 301-310 (1995).

\section{Acknowledgements}

We thank Jochen Mannhart for critically reading the manuscript and for the helpful comments, P. Specht and B. Stuhlhofer for the technical support on the Oxide MBE system, B. Stuhlhofer, Y. Link and S. Schmid for contact deposition, H. Hoier for XRR measurements and U. Salzberger for TEM specimen preparation. Y.W., W.S. and P.A.v.A. acknowledge funding from the European Union Seventh Framework Program (FP7/2007-2013) under grant agreement no. 312483 (ESTEEM2). 


\section{Author contributions}

F.B., G.L., G.G. and J.M. conceived the experiments. Samples were synthetized by F.B., G.L. and G.C.; atomic force microscopy and X-ray diffraction characterization were performed by F.B., electrical measurements by F.B., G.L. and G.C. Electron microscopy and related spectroscopy measurements were carried out by Y.W., W.S. and P.A.v.A. The initial draft was prepared by F.B, G.G. and J.M. All the authors contributed to the interpretation and discussion of the experimental data and to editing the initial draft.

\section{Additional information}

Supplementary Information accompanies this paper at http://www.nature.com/ naturecommunications

Competing financial interests: The authors declare no competing financial interests.
Reprints and permission information is available online at http://npg.nature.com/ reprintsandpermissions/

How to cite this article: Baiutti, F. et al. High-temperature superconductivity in space-charge regions of lanthanum cuprate induced by two-dimensional doping. Nat. Commun. 6:8586 doi: 10.1038/ncomms9586 (2015).

\section{(c) (1)}

This work is licensed under a Creative Commons Attribution 4.0 International License. The images or other third party material in this article are included in the article's Creative Commons license, unless indicated otherwise in the credit line; if the material is not included under the Creative Commons license, users will need to obtain permission from the license holder to reproduce the material. To view a copy of this license, visit http://creativecommons.org/licenses/by/4.0/ 